\title{
Fortaleza psicológica adolescente: relación con la inteligencia emocional y los valores
}

\section{Carlos Valiente Barroso}

Instituto Clínico y de Investigación Interdisciplinar en Neurociencias, España

mail: carlosvbsietedahotmail.com

ORCID: https://orcid.org/0000-0003-4670-0523

\section{Marta Arguedas-Morales}

Universidad Camilo José Cela, España

mail: arguedasmartalagmail.com

Orcid: https://orcid.org/0000-0003-2935-1878

\author{
Rafael Marcos Sánchez \\ Universidad Internacional de la Rioja, España \\ mail: rafael.marcos@unir.net \\ ORCID: https://orcid.org/0000-0002-3179-6211
}

\author{
Marta Martínez Vicente \\ Universidad Internacional Isabel I de Castilla, España \\ mail: marta.martinez.vicentedui1.es \\ ORCID: https://orcid.org/0000-0002-6601-9674
}

\section{RESUMEN}

Durante los últimos años, factores protectores de la salud mental como son la inteligencia emocional, los valores y un amplio espectro de variables de ajuste personal y psicológico, se han convertido en foco principal de investigación. Se plantea este estudio de metodología cuantitativa, no experimental transversal, descriptivo, inferencial y correlacional, cuyo objetivo general es comprobar las relaciones entre inteligencia emocional, los valores, la autoestima, el optimismo y tolerancia a la frustración en población adolescente. Se recogió información a través de una muestra de 161 estudiantes de Educación Secundaria de Madrid (España) $(M=14,51 ; D T=1,74)$. De los resultados obtenidos se observan relaciones positivas entre autoestima, optimismo y tolerancia a la frustración y las variables claridad emocional, reparación emocional, compromiso social y gratitud. Se confirma el carácter predictivo de gratitud y reconocimiento social en la autoestima y el optimismo, así como de reparación emocional en el optimismo y la tolerancia a la frustración. Se reafirma la necesidad de intervenciones programadas en educación emocional, como pieza clave del desarrollo del bienestar personal y psicológico y, en consecuencia, de una adecuada salud mental adolescente.

Palabras clave: inteligencia emocional, valores, autoestima, optimismo, frustración.

\section{Adolescent psychological strength: relationship to emotional intelligence and values}

\begin{abstract}
Protective factors of mental health, such as emotional intelligence, values, and a wide spectrum of variables pertaining to personal and psychological adjustment, have become a main focus for research in recent years. The general aim of this study, with its quantitative, non-experimental, cross-sectional, inferential and correlational methodology, was to verify the relationships between emotional intelligence, values, self-esteem, optimism and frustration tolerance in an adolescent population. Information was collected from a sample of 161 secondary education students in Madrid (Spain) (age in years: $M=14.51$; $S D=1.74$ ). From the results obtained, self-esteem, optimism and frustration tolerance were observed in positive relationships with the variables emotional clarity, emotional repair, social commitment and gratitude. Gratitude and social recognition were confirmed as predictors in self-esteem and optimism, as was emotional repair in optimism and frustration tolerance. The need for programmed interventions in emotional education is reaffirmed as a key element in the development of personal and psychological well-being and, consequently, of adequate mental health in adolescents.
\end{abstract}

Keywords: emotional intelligence, values, self-esteem, optimism, frustration. 


\section{Introducción}

Complementaria a la Psicología Clínica centrada en el estudio y tratamiento de los trastornos mentales, surge la Psicología Positiva, una nueva visión que presta atención a la promoción y prevención de la salud mental como un fin en sí mismo, es decir, a la experiencia del bienestar psicológico (Góngora y Casullo, 2009). Se trata de un enfoque orientado a mejorar la calidad de vida a partir del estudio del desarrollo de fortalezas, cualidades positivas y virtudes, enfatizando en las emociones positivas (Adler, 2017; Seligman 2014; Seligman y Csikszentmihalyi, 2000; Vásquez, 2013).

El constructo inteligencia emocional ha sido objeto de estudio desde la última década del siglo pasado cuando Mayer y Salovey (1997) lo definen como el conjunto de habilidades, a nivel intrapersonal e interpersonal, para percibir, expresar, comprender y regular las emociones, y que permiten, en el caso de los adolescentes, la promoción del bienestar y la mejora en el desarrollo de su ajuste psicológico (Mayer, Salovey y Caruso, 2004). Estas habilidades autopercibidas por las personas, se convierten en predictoras de estados emocionales asociados al buen ajuste del funcionamiento personal y social, influyendo en el desarrollo de conductas disfuncionales. Así, una inteligencia emocional alta se relacionaría con niveles mayores de bienestar personal, salud mental, ajuste psicológico, resolución de problemas sociales y de calidad de vida (Azpiazu, Esnaola y Sarasa, 2015; Peña-Sarrionandia, Mikolajczak y Gross, 2015; Pulido y Herrera, 2016).

A partir de esta consideración, estudios recientes identifican la inteligencia emocional a través de tres dimensiones que se corresponden con la percepción, comprensión y regulación emocional (Aritzeta et al., 2016), cobrando relevancia en los contextos sociales y educativos (Yudes, Peña y Pacheco et al., 2019), en los que es crucial abordar y afrontar circunstancias negativas. Por lo tanto, se configura como herramienta esencial en el abordaje de sucesos estresantes y como medio para conseguir el bienestar personal y emocional (Parhiala et al., 2018; Ruvalcaba, Gallegos, Solís, Gabriela y Bravo, 2019; Villanueva, Montoya-Castilla y Prado-Gascó, 2017).

Actualmente, no se duda de que la inteligencia emocional promueve eficientemente el afrontamiento del estrés, el bienestar emocional y, por lo tanto, el éxito personal e incluso profesional (Ordóñez, González, Montoya-Castilla, y Schoeps, 2014; Wang, Xie, y Cui, 2016). Así, se ha demostrado en estudios previos, en los que los estudiantes que poseen inteligencia emocional son capaces de percibir, utilizar, comprender y controlar sus emociones, presentando en menor grado desajustes psicológicos, estrés percibido, estrategias desadaptativas, ansiedad o depresión, conductas de riesgo y problemas de internalización; al mismo tiempo que, mejores estrategias de afrontamiento, relaciones interpersonales sanas y mayor satisfacción vital (Elipe, Mora-Merchán, Ortega-Ruiz y Casas, 2015; García, Quintana-Orts y Rey, 2020; Gómez-Baya, Mendoza, Paino y Matos, 2017; Resurrección, Salguero y Ruiz-Aranda, 2014; Rey, Extremera y Pena, 2011; Sánchez-Álvarez, Extremera y Fernández-Berrocal, 2016).

Los valores son metas motivacionales de distinta importancia, deseables y transituacionales, que sirven como principios vitales de la persona y constituyen creencias morales, duraderas y estables que conducen e impulsan las emociones, los pensamientos y el comportamiento (Peterson y Park, 2009; Reyes y Ferragut, 2016). Se diferencian, entre otros, los valores de poder, de conformidad, de tradición o de seguridad, que ligados a las preocupaciones propias de la persona, generan mayores niveles de ansiedad y menores de bienestar subjetivo. Estudios realizados al respecto indican que orientan la vida, e influyen a través del rol mediacional de las preocupaciones, en el bienestar personal. Relacionados con ellos, está la cultura como elemento diferenciador de la inteligencia emocional y las conductas sociales, que marca diferencias en los valores transmitidos y los patrones educativos determinantes en el desarrollo emocional (Alonso y Román, 2014; Gutiérrez y Expósito, 2015; Pulido y Herrera, 2016). Asimismo, pueden dificultar la integración a nivel socioeducativo (Pulido-Acosta y Herrera-Clavero, 2019), y, sabiendo que en la etapa adolescente se construye y consolida la personalidad, es importante recordar que el autoconocimiento, los comportamientos prosociales y el propio sistema de valores, forjan los cimientos emocionales (Ángel, 2020; Ferragut, Blanca y Ortiz-Tallo, 2014). La promoción y entrenamiento de los valores representa un elemento fundamental de la eficacia de intervenciones educativas, que, sustentadas desde la Psicología Positiva, han demostrado resultados positivos en la satisfacción vital y la felicidad de los más jóvenes (Linkis, Niemic, Gilham y Mayerson, 2014; Pulido-Acosta y Herrera-Clavero, 2020).

Por lo tanto, dentro de los programas de desarrollo socioemocional desarrollados en los últimos años, se incluye la formación en valores que se sustenta bajo el paradigma del modelo de desarrollo positivo adolescente; en el que adquieren una especial importancia las competencias sociales y emocionales ante el afrontamiento con éxito de la vida personal, así como en la adquisición de actitudes resilientes en esta población tan vulnerable (Pertegal, Oliva y Hernando, 2010, Oliva et al., 2010; Rodríguez-Fernández, Ramos-Díaz, Ros, Fernández-Zabala y Revuelta, 2016).

La autoestima juega un papel importante en el bienestar general, $y$, cuando es alta, deriva en beneficios para las personas al encontrarse bien consigo mismas Se asocia a mayor extraversión, autonomía, seguridad, conductas prosociales, desempeño positivo en grupo y mayor satisfacción en las relaciones interpersonales; siendo en caso contrario la promotora de inestabilidad, pesimismo o falta de autoconfianza. Es esencial ante los problemas y desafíos que las personas enfrentan en la vida, y desempeña un rol importante en el afrontamiento de situaciones estresantes, actuando como un almacén de sentimientos positivos (Rosenberg y Owens, 2001). Se han evidenciado los beneficios del desarrollo de las habilidades emocionales y de la autoestima en la prevención del desajuste psicológico de los adolescentes (Bleidorn et al., 2016; Carlo et al., 2014; Kökönyei et al., 2015; Reina y Oliva, 2015; Schoeps, Tamarit, González y Montoya-Castilla, 2019), identificándola como variable mediadora y explicativa del impacto de variables socioemocionales en dicho ajuste, lo que justifica su inclusión en estudios presentes y futuros (Kocayörük y Simsek, 2016; Thompson, Wojciak y Cooley, 2016).

El optimismo se ha considerado crucial en la adaptación de la persona, así como un predictor del bienestar y de la salud mental (Seligman 2014). Se define como la tendencia a conseguir resultados favorables en el futuro (Vásquez y Hervás, 2009) o el conjunto de expectativas positivas, que, asociadas a características disposicionales, median entre los acontecimientos externos y la interpretación subjetiva que la persona hace de ellos (Landa, Aguilar-Luzón y Salguero, 2008). Según Alarcón (2013), es la construcción de expectativas de eventos o acciones futuras positivas, en las que intervienen recursos cognitivos que analizan o emiten juicios de valor. Se explica desde dos perspectivas teóricas distintas pero no antagónicas; por un lado es una característica disposicional que se relaciona directamente con las expectativas, y bajo la cual, se esperan resultados positivos, aunque las circunstancias no sean las más favorables; por otro lado, es un estilo explicativo que parte del modelo de indefensión aprendida basado en la teoría atribucional, según la cual, las expectativas 
futuras están relacionadas con la explicación de hechos pasados. De acuerdo con esta perspectiva, una persona optimista tiende a explicar los acontecimientos negativos a través de atribuciones externas, específicas y temporales (Sánchez y Méndez, 2009). Por tanto, el optimismo permite dirigir la acción hacia la consecución de una meta, y explica las reacciones de afrontamiento de las personas ante sucesos negativos, siendo las más optimistas aquellas que atribuyen sus respuestas a la perseverancia, las aptitudes personales o el esfuerzo (Rodríguez y Castañeda, 2015; Seligman, 2004). Es un factor protector del bienestar y la salud emocional que permite manejar mejor del estrés, desarrollar relaciones interpersonales adecuadas y mayor fortaleza ante situaciones adversas, estresantes y traumáticas (Allan, Steger y Shin, 2013; González y Valdez, 2013; Jiang et al., 2014; Peters, Meevissen y Hanssen, 2013).

La frustración está asociada a objetivos inalcanzables y, en ella, la competencia social influye positivamente mediante una mayor tolerancia a la misma, facilitando la expresión de sentimientos positivos (dos Santos y Benevides, 2014). En sentido negativo, es generada por la insatisfacción de una meta o el bloqueo ante una situación, siendo mayor cuanto más intensa es la motivación (Ventura-León, Caycho-Rodríguez, Vargas-Tenazoa y Flores-Pino, 2018). Niveles altos de frustración se han vinculado con mayor irritabilidad, ira y agresividad (Rodríguez, Russa y Kircher, 2015; Sen y Mukherjee, 2014), por lo que su intolerancia se considera clave en el desarrollo de problemas emocionales, la competencia social, problemas psicopatológicos infantiles y de relaciones interpersonales, depresión, ansiedad y satisfacción con uno mismo (Jibeen, 2013; Mustaca, 2018; Perlman, Luna, Hein y Huppert, 2014). Así, la tolerancia a la frustración es la capacidad de resistencia de una persona ante acontecimientos adversos, estresantes o complicados ante los cuales retarda una respuesta, continuando a pesar de los obstáculos encontrados en el momento (Oliva, Pertegal et al., 2011). La baja tolerancia a la frustración implica una excesiva sensibilidad hacia todo, visibilizando siempre el lado negativo de cada situación, y, en el ámbito educativo, más que evitarla hay que preparar al estudiante para resistirla (Caballero, 2009).

Teniendo en cuenta lo anterior se plantea este estudio cuyo objetivo general es analizar las relaciones entre la inteligencia emocional, los valores, la autoestima, el optimismo y la tolerancia a la frustración en una muestra de estudiantes de secundaria. Se espera demostrar que existen diferencias significativas en los factores de inteligencia emocional y los valores adolescentes según distintos niveles (bajo, medio y alto) de autoestima, optimismo y tolerancia a la frustración; además del carácter predictivo de la inteligencia emocional y los valores en la autoestima, el optimismo y la tolerancia a la frustración.

\section{Método}

\section{Participantes}

En el estudio participaron 161 estudiantes de Educación Secundaria de un centro concertado de Madrid (España), seleccionados a través de un muestreo no probabilístico por conveniencia o incidental. Del total, un $34,78 \%$ cursaba $1^{\circ}(n=56)$, un 26,71 $\%$ cursaba $2^{\circ}(n=43)$, el $26,71 \%$ cursaba $3^{\circ}(n=43)$ y el $11,8 \%$ estaba en $4^{\circ}$ curso $(n=19)$. De edades entre los 12 y 18 años $(M$ $=14,51 ; D T=1,74)$, un $54,04 \%$ eran chicas $(n=87)$ y un $45,96 \%$ eran chicos $(n=74)$.

\section{Instrumentos}

Trait Meta-Mood Scale (TMMS; Salovey, Mayer, Goldman, Turvey y Palfai, 1995) en su adaptación español denominada Escala para la Evaluación de la Expresión, Manejo y Reconocimiento de Emociones TMMS-24 de Fernández-Berrocal, Extremera y Ramos (2004) evalúa la inteligencia emocional. Esta prueba de autoinforme contiene 24 ítems que aportan información independiente de los tres factores que la componen, en relación al grado en que cada uno percibe y atiende a sus propias emociones (Atención emocional), diferencia entre ellas (Claridad emocional) y es capaz de regularlas (Reparación emocional). Opciones de respuesta en escala Likert desde 1 (nada de acuerdo) hasta 5 (totalmente de acuerdo) y valores adecuados de consistencia interna (alpha de Cronbach) de 0,84 en atención; 0,82 en claridad y 0,81 en reparación.

Escala de Valores para Adolescentes (Oliva, Antolín et al., 2011) que evalúa la importancia que estudiantes adolescentes conceden a un conjunto de valores implicados en su desarrollo positivo. Está compuesta por 24 ítems en formato tipo Likert con respuestas desde 1 (nada importante) hasta 7 (lo más importante) y permite obtener puntuaciones parciales en compromiso social, prosocialidad, justicia e igualdad, honestidad, integridad, responsabilidad, reconocimiento social y hedonismo, que son las incluidas en este estudio, así como en tres subescalas globales (valores sociales, valores personales y valores individualistas). La fiabilidad del instrumento medida a través del índice de consistencia interna (alpha de Cronbach) es de 0,89.

Cuestionario de Gratitud GQ-6 (McCullough, Emmons y Tsang, 2002) traducido por Beléndez (2011), se compone de 6 ítems (p ej., "Me siento agradecido/a a una gran cantidad de gente") que evalúan la tendencia a sentir gratitud en la vida cotidiana. Cuenta con un coeficiente alfa de 0.84 .

Escala de Autoestima (Rosenberg, 1965), como prueba unidimensional que consta de 10 ítems (p ej., "A veces me siento realmente inútil"), evaluando la autoestima global de los adolescentes. Escala tipo Likert desde 1 (totalmente en desacuerdo) hasta 4 (totalmente de acuerdo) cuyo índice de fiabilidad (alpha de Cronbach) es de 0,92.

Escala para la Evaluación del Optimismo (Bar-On y Parker, 2000) incluida en la versión extensa del Emotional Quotient Inventory Youth Version (EQ-i: YV), desarrollado para evaluar la inteligencia socioemocional en su adaptación española (Oliva, Antolín et al., 2011). Evalúa la capacidad del sujeto para mantener actitudes positivas y sentirse satisfecho con uno mismo y los demás, es decir, se valora el optimismo y la felicidad del sujeto. Consta de ocho ítems (p ej., "Me siento seguro de mí mismo) que deben ser puntuados en una escala tipo Likert de cinco puntos desde 1 (nunca) hasta 5 (siempre). Consistencia interna (alpha de Cronbach) de 0,91.

Escala para la Evaluación de la Tolerancia a la Frustración (BarOn y Parker, 2000) en su versión española (Oliva, Antolín et al., 2011) inicialmente desarrollada para evaluar la inteligencia socioemocional y que mide la capacidad para manejar y resistir situaciones de estrés y el control de los impulsos. Consta de ocho ítems (p ej., "Me resulta difícil controlar mi ira") de respuesta Likert de cinco puntos desde 1 (nunca) hasta 5 (siempre). Índice de consistencia interna adecuado medido a través del coeficiente alpha de Cronbach de 0,77.

\section{Procedimiento}

Después de informar y pedir la autorización y el consentimiento tanto a la dirección del centro como a las familias, se procedió a la aplicación de los cuestionarios a todo el alumnado en una sesión de clase (duración aproximada 60 minutos), en pre- 
sencia del tutor y uno de los miembros del equipo investigador que, además, forma parte del equipo docente del centro. Se aseguró en todo momento el anonimato y la confidencialidad de los resultados obtenidos, así como la participación voluntaria con el fin de reducir sesgos en las respuestas que pudiesen dificultar el posterior análisis y la validez de los resultados obtenidos. Con la información recogida se diseñó un estudio de metodología cuantitativa, no experimental, transversal, inferencial y correlacional.

\section{Análisis estadísticos}

Se calcularon las correlaciones de las variables (bivariadas de Pearson) y se realizaron una serie de análisis diferenciales a través de la prueba estadística de análisis univariante de la varianza (ANOVA), calculando el estadístico $F$ de Snedecor y como contraste post hoc la corrección de Scheffé con valor de significación de .05. Se tomaron como factores independientes la autoestima, el optimismo y la tolerancia a la frustración, y como variables dependientes la inteligencia emocional (atención, claridad y reparación emocional) y los valores adolescentes (compromiso social, prosocialidad, justicia, honestidad, integridad, responsabilidad, reconocimiento social, hedonismo y gratitud). Se formaron tres grupos en cada variable independiente (autoestima, optimismo y tolerancia a la frustración) a partir del cálculo previo de los percentiles 33 y 67 quedando conformados en cada caso los grupos de nivel bajo (percentiles inferiores al 33), nivel medio (percentiles desde el 33 hasta el 67, ambos incluidos) y nivel alto (percentiles superiores al 67).

Por último, se realizaron tres análisis de regresión (método Stepwise) para determinar el valor predictivo de las variables vinculadas a la inteligencia emocional y los valores sociales en la autoestima, el optimismo y la tolerancia a la frustración. Los análisis de datos se realizaron a través del programa SPSS en su versión 25.0 para Windows.

\section{Resultados}

\section{Correlaciones entre las variables del estudio}

Los resultados del análisis correlacional que aparecen en la Tabla 1 muestran relaciones significativas y positivas (aunque moderadas) entre autoestima y claridad emocional, reparación emocional, compromiso social, honestidad, reconocimiento social y gratitud. Asimismo, optimismo se relaciona significativa y positivamente con claridad emocional, reparación emocional, compromiso social, justicia e igualdad, honestidad, integridad, responsabilidad, reconocimiento social y gratitud. Por último, tolerancia a la frustración mantiene relaciones significativas y positivas con claridad emocional, reparación emocional, compromiso social, prosocialidad, justicia e igualdad, responsabilidad y gratitud.

Tabla 1

Correlaciones bivariadas de Pearson entre las variables del estudio

\begin{tabular}{lccc}
\hline & Autoestima & Optimismo & $\begin{array}{c}\text { Tolerancia a la } \\
\text { frustración }\end{array}$ \\
\hline Atención emocional & 0,113 & 0,007 & $-0,153$ \\
Claridad emocional & $0,174^{*}$ & $0,312^{* *}$ & $0,228^{*}$ \\
Reparación emocional & $0,204^{* *}$ & $0,397^{* *}$ & $0,365^{* *}$ \\
Compromiso social & $0,160^{*}$ & $0,245^{* *}$ & $0,185^{* *}$ \\
Prosocialidad & 0,118 & 0,135 & $0,252^{* *}$ \\
Justicia e igualdad & 0,126 & $0,194^{*}$ & $0,210^{* *}$ \\
Honestidad & $0,176^{*}$ & $0,264^{* *}$ & 0,076
\end{tabular}

\begin{tabular}{llll} 
Integridad & 0,151 & $0,215^{* *}$ & 0,135 \\
Responsabilidad & 0,123 & $0,267^{* *}$ & $0,330^{* *}$ \\
Reconocimiento social & $0,164^{*}$ & $0,208^{* *}$ & $-0,145$ \\
Hedonismo & 0,143 & 0,138 & $-0,067$ \\
Gratitud & $0,406^{* *}$ & $0,357^{* *}$ & $0,218^{* *}$ \\
\hline
\end{tabular}

${ }^{*} p<0,05 ;{ }^{* *} p<0,01$

Inteligencia emocional y valores: diferencias en función de la autoestima, Inteligencia el optimismo y la tolerancia a la frustración

Los resultados del análisis de la inteligencia emocional y los valores según distintos niveles de autoestima, indican diferencias significativas en claridad emocional y gratitud (ver Tabla 2). Al realizar los contrastes post hoc se comprueban diferencias significativas entre los grupos de autoestima alta y media en claridad emocional y entre los grupos de alta autoestima y los de media y baja en gratitud. Aunque no se encuentran diferencias significativas en el resto de variables, se observan en todas ellas valores medios mayores en el grupo de autoestima alta.

Tabla 2

Análisis de la varianza de la inteligencia emocional y los valores en función de la autoestima

\begin{tabular}{|c|c|c|c|c|c|}
\hline \multirow[b]{2}{*}{ Variables } & \multicolumn{3}{|c|}{ Rango de medias $(D T)$} & \multirow[b]{2}{*}{$F(2,157)$} & \multirow[b]{2}{*}{ Post-hoc } \\
\hline & $\begin{array}{c}\text { Autoestima } \\
\text { baja } \\
(n=54)\end{array}$ & $\begin{array}{c}\text { Autoestima } \\
\text { media } \\
(n=64)\end{array}$ & $\begin{array}{c}\text { Autoestima } \\
\text { alta } \\
(n=43)\end{array}$ & & \\
\hline $\begin{array}{l}\text { Atención } \\
\text { emocional }\end{array}$ & $24,25(8,33)$ & $23,92(6,64)$ & $25,16(6,49)$ & 0,38 & \\
\hline $\begin{array}{l}\text { Claridad } \\
\text { emocional }\end{array}$ & $24,69(7,27)$ & $23,73(6,11)$ & $27,37(5,71)$ & $4,10^{*}$ & $2<3^{*}$ \\
\hline $\begin{array}{l}\text { Reparación } \\
\text { emocional }\end{array}$ & $26,62(7,24)$ & $25,90(6,38)$ & $28,69(6,54)$ & 2,23 & \\
\hline $\begin{array}{l}\text { Compromiso } \\
\text { social }\end{array}$ & $12,71(4,18)$ & $12,74(3,72)$ & $13,16(4,00)$ & 0,17 & \\
\hline Prosocialidad & $14,71(3,88)$ & $13,90(3,51)$ & $14,73(3,64)$ & 0,92 & \\
\hline $\begin{array}{l}\text { Justicia e } \\
\text { igualdad }\end{array}$ & $16,56(3,96)$ & $16,00(3,77)$ & $17,17(3,17)$ & 1,25 & \\
\hline Honestidad & $18,05(3,52)$ & $17,91(2,77)$ & $18,42(3,01)$ & 0,33 & \\
\hline Integridad & $15,26(3,99)$ & $15,14(3,48)$ & $15,95(3,40)$ & 0,65 & \\
\hline $\begin{array}{l}\text { Responsabi- } \\
\text { lidad }\end{array}$ & $15,54(3,97)$ & $15,34(3,32)$ & $15,64(4,22)$ & 0,08 & \\
\hline $\begin{array}{l}\text { Reconoci- } \\
\text { miento social }\end{array}$ & $10,33(5,78)$ & $11,60(4,82)$ & $12,07(4,97)$ & 1,45 & \\
\hline Hedonismo & $16,90(6,76)$ & $18,47(6,63)$ & $18,02(7,88)$ & 0,74 & \\
\hline Gratitud & $24,97(6,86)$ & $32,09(9,65)$ & $34,33(11,69)$ & $13,40^{* * *}$ & $1<2^{* * *} ; 1<3^{* * *}$ \\
\hline
\end{tabular}

En el segundo análisis de varianza con el optimismo como variable independiente, se observan diferencias significativas en claridad emocional, reparación emocional, honestidad, integridad, responsabilidad y gratitud. Se comprueba en el análisis post hoc, tal y como se muestra en la tabla 3, que tales diferencias se mantienen entre varios grupos de optimismo. 
Tabla 3.

Análisis de la varianza de la inteligencia emocional y los valores en función del optimismo

\begin{tabular}{|c|c|c|c|c|c|}
\hline \multirow[b]{2}{*}{ Variables } & \multicolumn{3}{|c|}{ Rango de medias $(D T)$} & \multirow[b]{2}{*}{$F(2,158)$} & \multirow[b]{2}{*}{ Post-hoc } \\
\hline & $\begin{array}{c}\text { Optimismo } \\
\text { bajo } \\
(n=60)\end{array}$ & $\begin{array}{c}\text { Optimismo } \\
\text { medio } \\
(n=52)\end{array}$ & $\begin{array}{c}\text { Optimismo } \\
\text { alto } \\
(n=49)\end{array}$ & & \\
\hline $\begin{array}{l}\text { Atención } \\
\text { emocional }\end{array}$ & $24,47(7,76)$ & $24,69(5,73)$ & $23,79(7,87)$ & 0,21 & \\
\hline $\begin{array}{l}\text { Claridad } \\
\text { emocional }\end{array}$ & $22,38(6,90)$ & $25,13(4,74)$ & $27,98(6,55)$ & $11,01^{* * *}$ & $1<3^{* *}$ \\
\hline $\begin{array}{l}\text { Reparación } \\
\text { emocional }\end{array}$ & $23,43(6,41)$ & $27,40(5,47)$ & $30,33(6,82)$ & $16,58^{* * *}$ & $1<2^{* *} ; 1<3^{* * *}$ \\
\hline $\begin{array}{l}\text { Compromiso } \\
\text { social }\end{array}$ & $12,06(3,96)$ & $12,98(3,55)$ & $13,66(4,16)$ & 2,24 & \\
\hline Prosocialidad & $13,86(3,57)$ & $14,33(3,55)$ & $15,16(3,85)$ & 1,64 & \\
\hline $\begin{array}{l}\text { Justicia e } \\
\text { igualdad }\end{array}$ & $16,03(3,93)$ & $16,52(3,38)$ & $17,02(3,70)$ & 0,93 & \\
\hline Honestidad & $17,58(3,59)$ & $17,84(2,77)$ & $19,08(2,46)$ & $3,42^{*}$ & $1<3^{*}$ \\
\hline Integridad & $14,96(3,85)$ & $14,82(3,50)$ & $16,56(3,23)$ & $3,56^{*}$ & \\
\hline Responsabilidad & $14,35(3,95)$ & $15,82(3,38)$ & $16,55(3,59)$ & $4,99^{* *}$ & $1<3^{*}$ \\
\hline $\begin{array}{l}\text { Reconocimiento } \\
\text { social }\end{array}$ & $10,73(5,46)$ & $10,92(4,77)$ & $12,28(5,39)$ & 1,29 & \\
\hline Hedonismo & $17,35(7,64)$ & $17,54(6,51)$ & $18,60(6,71$ & 0,46 & \\
\hline Gratitud & $26,10(7,78)$ & $30,77(5,91)$ & $35,04(13,70)$ & $11,87^{* * *}$ & $1<2^{*} ; 1<3^{* * *}$ \\
\hline
\end{tabular}

${ }^{*} p<0,05 ;{ }^{* *} p<0,01 ;{ }^{* * *} p<0,001$

Un tercer análisis diferencial según los niveles de tolerancia a la frustración informa de diferencias significativas en atención emocional, claridad emocional, reparación emocional, prosocialidad, responsabilidad y gratitud. Estas diferencias, según la prueba post hoc, se establecen principalmente entre los grupos de nivel bajo y alto de tolerancia a la frustración (Ver Tabla 4).

Tabla 4.

Análisis de la varianza de la inteligencia emocional y los valores en función de la tolerancia a la frustración

\begin{tabular}{|c|c|c|c|c|c|}
\hline \multirow[b]{2}{*}{ Variables } & \multicolumn{3}{|c|}{ Rango de medias $(D T)$} & \multirow[b]{2}{*}{$F(2,158)$} & \multirow[b]{2}{*}{ post-hoc } \\
\hline & $\begin{array}{c}\text { Tolerancia } \\
\text { baja } \\
(n=60)\end{array}$ & $\begin{array}{c}\text { Tolerancia } \\
\text { media } \\
(n=52)\end{array}$ & $\begin{array}{c}\text { Tolerancia } \\
\text { alta } \\
(n=48)\end{array}$ & & \\
\hline $\begin{array}{l}\text { Atención } \\
\text { emocional }\end{array}$ & $26,41(7,40)$ & $23,84(6,91)$ & $22,61(6,76)$ & $3,859^{*}$ & $1>3^{*}$ \\
\hline $\begin{array}{l}\text { Claridad } \\
\text { emocional }\end{array}$ & $24,12(6,73)$ & $23,75(6,69)$ & $27,40(5,57)$ & $4,912^{* *}$ & $\begin{array}{l}1<3^{*} \\
2<3^{*}\end{array}$ \\
\hline $\begin{array}{l}\text { Reparación } \\
\text { emocional }\end{array}$ & $24,59(6,94)$ & $26,47(6,52)$ & $29,72(6,15)$ & $7,780^{* * *}$ & $1<3^{* * *}$ \\
\hline $\begin{array}{l}\text { Compromiso } \\
\text { social }\end{array}$ & $12,02(4,39)$ & $13,07(3,76)$ & $13,47(3,44)$ & 1,880 & \\
\hline Prosocialidad & $13,97(3,84)$ & $13,80(3,50)$ & $15,60(3,41)$ & $3,738^{*}$ & $2<3^{*}$ \\
\hline $\begin{array}{l}\text { Justicia e } \\
\text { igualdad }\end{array}$ & $16,03(4,14)$ & $16,43(3,68)$ & $17,06(3,08)$ & 0,969 & \\
\hline Honestidad & $18,62(3,67)$ & $17,23(2,67)$ & $18,54(2,59)$ & $3,596^{*}$ & \\
\hline
\end{tabular}

\begin{tabular}{|c|c|c|c|c|}
\hline Integridad & $15,12(3,90)$ & $15,21(3,57)$ & $15,91(3,35)$ & 0,685 \\
\hline $\begin{array}{l}\text { Responsabili- } \\
\text { dad }\end{array}$ & - $14,53(4,36)$ & $15,32(3,15)$ & $16,73(3,40)$ & $4,507^{*}$ \\
\hline $\begin{array}{l}\text { Reconocimien- } \\
\text { to social }\end{array}$ & $12,03(5,37)$ & $11,64(4,92)$ & $9,82(5,27)$ & 2,522 \\
\hline Hedonismo & $18,98(7,76)$ & $17,33(7,42)$ & $16,93(5,24)$ & 1,250 \\
\hline Gratitud & $29,87(10,83)$ & $28,11(7,15)$ & $33,54(11,74)$ & $13,400^{* *}$ \\
\hline
\end{tabular}

Capacidad predictiva de la inteligencia emocional y los valores en la autoestima, el optimismo y la tolerancia a la frustración

El primer análisis de regresión (Tabla 5) indica que la autoestima se encuentra predicha por dos variables, gratitud $(b=0,425$; $t=5,844 ; p<0,001)$ y reconocimiento social $(b=0,197 ; t=2,717 ; p$ $<0,01)$ explicando juntas el 19,6\% de la varianza y contribuyendo significativamente a la explicación de la autoestima.

Tabla 5.

Resultados del análisis de regresión con la variable autoestima como variable criterio y las vinculadas a la inteligencia emocional, los valores y la gratitud como variables predictoras

\begin{tabular}{lccccc}
\hline Modelo & $R$ & $R^{2}$ & $R^{2}$ ajustado & $F(\mathrm{gl})$ & $p$ \\
\cline { 1 - 4 } Modelo 1 $^{\mathrm{a}}$ & 0,410 & 0,168 & 0,162 & $30,674(1,153)$ & 0,000 \\
Modelo 2 $^{\mathrm{b}}$ & 0,455 & 0,207 & 0,196 & $19,671(2,153)$ & 0,000 \\
\cline { 1 - 2 } & &
\end{tabular}

En la Tabla 6, aparecen los resultados del segundo análisis de regresión que indican que la variable optimismo se encuentra predicha por tres variables, reparación emocional $(b=0,287$; $t=3,879 ; p<0,001)$, gratitud $(b=0,292 ; t=3,943 ; p<0,001)$ y reconocimiento social $(b=0,240 ; t=3,427 ; p<0,001)$ explicando significativamente el $25,9 \%$ de la varianza total.

Tabla 6.

Resultados del análisis de regresión con la variable optimismo como variable criterio y las vinculadas a la inteligencia emocional, los valores y la gratitud como variables predictoras

\begin{tabular}{lccccc}
\hline Modelo & $R$ & $R^{2}$ & $R^{2}$ ajustado & $F(\mathrm{gl})$ & $p$ \\
\hline Modelo 1 $^{\mathrm{a}}$ & 0,388 & 0,151 & 0,145 & $26,769(1,152)$ & 0,000 \\
Modelo 2 $^{\mathrm{b}}$ & 0,465 & 0,216 & 0,205 & $20,652(2,152)$ & 0,000 \\
Modelo 3 $^{\mathrm{c}}$ & 0,523 & 0,273 & 0,259 & $18,668(3,152)$ & 0,000 \\
\hline
\end{tabular}

${ }^{\text {a }}$ Reparación emocional

${ }^{\mathrm{b}}$ Reparación emocional, gratitud

c Reparación emocional, gratitud, reconocimiento social

El último análisis de regresión arroja los resultados que aparecen en la Tabla 7 y en la que aparecen dos variables predictoras de la tolerancia a la frustración. En el modelo 2, se incluyen las variables reparación emocional $(b=0,294 ; t=3,690 ; p<0,001) \mathrm{y}$ responsabilidad $(b=0,213 ; t=2,676 ; p<0,01)$ explicando conjuntamente el $17,1 \%$ de la varianza total. 
Tabla 7 .

Resultados del análisis de regresión con la variable tolerancia a la frustración como variable criterio y las vinculadas a la inteligencia emocional y valores como variables predictoras

\begin{tabular}{lccccc}
\hline Modelo & $R$ & $R^{2}$ & $R^{2}$ ajustado & $F(\mathrm{gl})$ & $P$ \\
\hline $\begin{array}{l}\text { Modelo } \\
1^{\text {a }}\end{array}$ & 0,378 & 0,143 & 0,137 & $25,546(1,154)$ & 0,000 \\
$\begin{array}{l}\text { Modelo } \\
2^{\text {b }}\end{array}$ & 0,426 & 0,182 & 0,171 & $16,860(2,154)$ & 0,000 \\
\hline
\end{tabular}

a Reparación emocional

${ }^{\mathrm{b}}$ Reparación emocional, responsabilidad

\section{Discusión y conclusiones}

En la línea de estudios que señalan los beneficios de la inteligencia emocional y social en el desarrollo personal, el bienestar psicológico y la salud mental del discente, se propone el presente estudio cuyo objetivo es comprobar las relaciones entre la inteligencia emocional y los valores con variables vinculadas al bienestar psicológico como son la autoestima, el optimismo y la tolerancia a la frustración en una muestra de adolescentes españoles.

Se ha comprobado que cuanto mayor es la claridad emocional, reparación emocional, compromiso social y gratitud ante la vida, más elevada es la autoestima, el optimismo y la tolerancia a la frustración. Al mismo tiempo, valores como la honestidad y el reconocimiento social son mayores cuando lo son también la autoestima y el optimismo. La responsabilidad y el valor de justicia e igualdad se relacionan directamente con el optimismo y la tolerancia a la frustración, variable esta última que mantiene relaciones directas con las conductas prosociales de los adolescentes. Entre estos, los más optimistas son los que informan de mayor integridad. Estos resultados están en consonancia con otros que evidencian que los adolescentes que diferencian y comprenden sus emociones pueden sentir afectos positivos y, por tanto, mayor satisfacción vital en sentido global (Gómez-Baya et al., 2017; Rey et al., 2011; Sánchez-Álvarez et al., 2016). Al tener mayores niveles de inteligencia emocional a través de sus habilidades para percibir, comprender y regular las propias emociones, presentan menores desajustes en su bienestar (Elipe et al., 2015; García et al., 2020). Así, se justifica porque las personas con altos niveles de inteligencia emocional se caracterizan por emplear un razonamiento flexible, algo que propicia una mayor comprensión y actitudes de ayuda hacia los demás, influyendo al tiempo en la mejora de la integración y bienestar social (Jiménez y López-Zafra, 2013; Pulido-Acosta y Herrera-Clavero, 2019).

Se han encontrado diferencias significativas en claridad emocional y gratitud entre los adolescentes con bajo y alto nivel de autoestima, optimismo y tolerancia a la frustración. Como era previsible, los adolescentes con mayores niveles de optimismo y tolerancia a la frustración son las que puntúan más alto en reparación emocional y responsabilidad. Asimismo, mayores niveles de tolerancia a la frustración se identifican con mayor atención a las emociones y conductas prosociales; y niveles altos de optimismo a mayor honestidad e integridad. Las personas optimistas perciben con menor gravedad los problemas y el riesgo de problemas futuros, $y$, además, frente a las dificultades actúan con más eficacia (González y Valdez, 2013; Rodríguez y Castañeda, 2015), lo que está en consonancia con la visión del optimismo como expectativa generalizada y global que se relaciona con niveles de satisfacción vital generales, realización personal, estilos atribucionales, personalidad y felicidad subjetiva (Jiang et al.,
2014; Marrero y Carballeira, 2010; Pulido-Acosta y Herrera-Clavero, 2020).

Por último, cabe destacar, tras los análisis de regresión efectuados, que es previsible que la gratitud y el reconocimiento social ejerzan un efecto positivo tanto en la autoestima como en el optimismo. Se demuestra el carácter predictivo del factor reparación emocional en el optimismo y la tolerancia a la frustración, y como ésta es a su vez predicha también por la responsabilidad. Resultados en consonancia con otros previos que demuestran que la gratitud actúa como barrera de las emociones negativas, incrementa el optimismo y promueve el bienestar personal (Allan et al., 2013; Peters et al., 2013).

Con todo ello, estos resultados refuerzan estudios anteriores que informan que los jóvenes con alta autoestima muestran mayor adaptación a eventos vitales estresantes (Kocayörük y Şimşek, 2016; Thompson et al., 2016); aquellos con baja autoestima presentan mayor riesgo en su salud mental y bienestar emocional (von Soest, Wichstrøm y Kvalem, 2016), siendo determinante del desajuste psicológico en los adolescentes (Keane y Loades, 2016; Reina y Oliva, 2015; Schoeps et al., 2019). La valoración que cada uno hace de sí mismo, junto con las habilidades de percepción, comprensión y regulación emocional reduce los síntomas emocionales y las conductas disfuncionales, ayudando en la adaptación a nivel físico y psicológica de la adolescencia (Bleidorn et al., 2016; Carlo et al., 2014; Kökönyei et al., 2015).

Las competencias emocionales se relacionan directamente con la autoestima e inversamente con los problemas emocionales y conductuales, convirtiéndose por lo tanto en indicadores de un buen ajuste psicológico, aunque sean constructos que describan procesos cognitivos y emocionales diferentes (Cheung et al., 2015; Ordóñez, Maganto y González., 2015; Schoeps et al., 2019; Reina y Oliva, 2015). Por ello, se proclama la necesidad de programar intervenciones educativas adecuadas, ya que, como se señala en la literatura previa, el ajuste psicológico puede mejorarse mediante el desarrollo de estrategias de comprensión y regulación de las emociones. Los adolescentes controlarán mejor sus fuentes de estrés si gestionan sus impulsos emocionales, potenciando las emociones positivas y reduciendo las negativas, de manera que se adaptan mejor a los cambios y las adversidades y, por ello, el impacto en la salud mental es positivo (Anto y Jayan, 2016). La expresión de sus preocupaciones y miedos es indicativo de una mayor percepción emocional (Mayer, Caruso y Salovey, 2016).

Se evidencia la importancia de la gratitud como fortaleza en los adolescentes a través de su relación directa con la autoestima, el optimismo y la tolerancia a la frustración, actuando como factor protector ante el estrés y la adversidad, estableciendo una relación directa con mayor satisfacción vital, bienestar subjetivo y calidad de vida (Ángel, 2020; Ferragut et al., 2014; Giménez, Vázquez y Hervás, 2010; Linkis et al., 2014; Reyes y Ferragut, 2016). La intervención en valores mejora significativamente el ajuste personal reduciendo sentimientos negativos como la culpabilidad y la timidez, pero, además, incrementan la seguridad y la confianza en uno mismo, estimulando actitudes de tolerancia, búsqueda de soluciones, afrontamiento y superación de adversidades; valores sobre los que se construyen y desarrollan proyectos de vida personales, sociales y psicológicos saludables (De Mendoza, Medina y Hernández, 2003).

Este estudio no está exento de limitaciones. Por un lado, su carácter transversal impide tener una visión completa de la evolución de los estudiantes en las variables analizadas en distintos momentos, además de no poder determinar relaciones causales entre ellas, estableciéndose únicamente relaciones de correlación. Por lo tanto, en futuras investigaciones será interesante realizar estudios longitudinales para comprobar si las diferencias se 
mantienen a lo largo del tiempo. Por otro lado, la información se recoge a través de autoinformes que, a su vez, podrían ser complementados con pruebas de carácter objetivo. El tamaño reducido y la procedencia de la muestra, al no ser aleatoria y limitarse a un solo centro educativo, no permite la generalización de los resultados. Además, el amplio rango de edad de los estudiantes (entre 12 y 18 años) puede ser motivo de mayor variabilidad y sesgo en los resultados, por lo que en futuros trabajos podrían considerarse rangos de edad menos amplios y comparar así los resultados.

No obstante, a pesar de las limitaciones comentadas, y dado que la literatura ha demostrado las implicaciones prácticas que tiene la educación emocional y el desarrollo de las competencias socioemocionales desde los primeros niveles de escolarización (Alonso, Castedo y Roales, 2015; Ruiz-Aranda et al., 2013), siendo especialmente necesaria en la etapa adolescente, es preciso seguir realizando estudios que aporten información relevante tanto a investigadores como a profesionales de la enseñanza para conseguir un mayor ajuste psicológico, el incremento de los sentimientos de bienestar, de satisfacción vital y, por consiguiente, adecuados niveles de bienestar y salud mental.

\section{Referencias}

Adler, A. (2017). Educación positiva: Educando para el éxito académico y para la vida plena. Papeles del Psicólogo, 38(1), 50-57. https:/ / doi.org/10.23923/pap.psicol2017.2821

Alarcón, R. (2013). Construcción y validación de una escala para medir el optimismo. En J. C. Alchieri y J. Barreiros (Org.). Conferências do XXXIV Congresso Interamericano de Psicología. (pp. 377-389). SBPOT.

Allan, B. A., Steger, M.F., y Shin, J.Y. (2013). Thanks? Gratitude and well-being over the Thanksgiving holiday among college students. The Journal of Positive Psychology, 8, 91-102.

Alonso, J. D., Castedo, A. L., y Roales, E. Á. (2015). Implicación de variables sociales y educativas en la conducta asertiva adolescente. Aula Abierta, 43(1), 26-31. https:/ / doi.org/10.1016/j. aula.2014.05.004

Alonso, J. y Román, J. M. (2014). Nivel sociocultural, prácticas educativas familiares y autoestima de los hijos en edades tempranas. Revista de Investigación Educativa, 32, 187-202. https://doi.org/10.6018/rie.32.1.173421

Ángel, N. G. (2020). Inteligencia emocional percibida en estudiantes de educación superior: análisis de las diferencias en las distintas dimensiones. Actualidades en Psicología, 34(128), $17-$ 33. https://doi.org/10.15517/ap.v34i128.34469

Anto, S. P., y Jayan, C. (2016). Self-esteem and emotion regulation as determinants of mental health of youth. Journal of Projective Psychology \& Mental Health, 23(1), 34-40.

Aritzeta, A., Balluerka, N., Gorostiaga, A., Alonso-Arbiol, I., Haranburu, M., y Gartzia, L. (2016). Classroom emotional intelligence and its relationship with school performance. European Journal of Education and Psychology, 9(1), 1-8.

Azpiazu, L., Esnaola, I., y Sarasa, M. (2015). Predictive capacity of social support on emotional intelligence in adolescence. European Journal of Education and Psychology, 8(1), 23-29.

Beléndez, M. (2011). Traducción y adaptación al castellano del cuestionario de Gratitud Disposicional (CG-6). Universidad de Alicante. http://local.psy.miami.edu/faculty/mmccullough/Gratitude-Related\%20Stuff/Spanish_gratitude_scale_Belendez2011.pdf

Bleidorn, W., Arslan, R. C., Denissen, J. J. A., Rentfrow, P. J., Gebauer, J. E., Potter, J., y Gosling, S. D. (2016). Age and gender differences in self-esteem - A cross-cultural window. Journal of Personality and Social Psychology, 111(3), 396-410. https://doi. org /10.1037/pspp0000078

Caballero, A. L. (2009). La frustración como elemento educativo. Padres y Maestros, 323, 24-29.

Carlo, G., Mestre, M. V., McGinley, M. M., Tur-Porcar, A., Samper, P., y Opal, D. (2014). The protective role of prosocial behaviors on antisocial behaviors: The mediating effects of deviant peer affiliation. Journal of Adolescence, 37(4), 359-366. https: / / doi. org/10.1016/j.adolescence.2014.02.009

Cheung, C. K., Cheung, H. Y., y Hue, M. T. (2015). Emotional intelligence as a basis for self-esteem in young adults. The Journal of Psychology: Interdisciplinary and Applied, 149(1), 63-84. https:/ / doi.org/10.1080/00223980.2013.838540

De Mendoza, R. A., Medina, R. D., y Hernández, P. H. (2003). Evaluación de la mejora en valores de realización personal y social en adolescentes que han participado en un programa de intervención. Psicothema, 15(4), 589-594.

Dos Santos, T. V., y Benevides, A. (2014). Habilidades sociales y frustración en estudiantes de medicina. Ciencias Psicológicas, 8(2), 163-172.

Elipe, P., Mora-Merchán, J. A., Ortega-Ruiz, R., y Casas, J. A. (2015). Perceived emotional intelligence as a moderator variable between cybervictimization and its emotional impact. Frontiers in Psychology, 6, 486. https:/ / doi.org/10.3389/fpsyg.2015.00486

Fernández-Berrocal, P., Extremera, N., y Ramos, N. (2004). Validity and reliability of the spanish modified version of the Trait Meta-Mood Scale. Psychological Reports, 94(3), 751-755. https://doi.org/10.2466/pr0.94.3.751-755

Ferragut, M., Blanca, M. J. y Ortiz-Tallo, M. (2014). Analysis of adolescent profiles by gender: strenghts, attitudes toward violence and sexism. Spanish Journal of Psychology, 17, 1-10. https:/ / doi.org/10.1017/sjp.2014.60

García, L., Quintana-Orts, C., y Rey, L. (2020). Cibervictimización y satisfacción vital en adolescentes: la inteligencia emocional como variable mediadora. Revista de Psicología Clínica con Niños y Adolescentes, 7(1), 38-45. https://doi.org/ 10.21134/rpcna.2020.07.1.5

González, N. I., y Valdez, J. L. (2013). Optimismo: validación de una escala para adolescentes mexicanos. Revista Peruana de Psicología y Trabajo Social, 2(1), 121-130.

Giménez, M., Vázquez, C., y Hervás, G. (2010). El análisis de las fortalezas psicológicas en la adolescencia. Más allá de los modelos de vulnerabilidad. Psychology, Society EEducation, 2, 97-116.

Gomez-Baya, D., Mendoza, R., Paino, S., y de Matos, M. G. (2017). Perceived emotional intelligence as a predictor of depressive symptoms during mid-adolescence: A two-year longitudinal study on gender differences. Personality and individual differences, 104, 303-312. https:/ / doi.org/10.1016/j.paid.2016.08.022

Góngora, V. C., y Casullo, M. M. (2009). Factores protectores de la salud mental: Un estudio comparativo sobre valores, autoestima e inteligencia emocional en población clínica y población general. Interdisciplinaria, 26(2), 183-205.

Gutiérrez, M. y Expósito, J. (2015). Autoconcepto, dificultades interpersonales, habilidades sociales y conductas asertivas en adolescentes. Revista Española de Orientación y Psicopedagogía, 26(2), 42-58. https://doi.org/10.5944/reop.vol.26. num.2.2015.15215

Keane, L., y Loades, M. (2016). Review: Low self-esteem and internalizing disorders in young people - a systematic review. Child and Adolescent Mental Health, 22(1), 4-15. https:/ /doi. org/10.1111/camh.12204

Kocayörük, E., y Şimşek, Ö. F. (2016). Parental Attachment and Adolescents' Perception of School Alienation: The Mediation 
Role of Self-Esteem and Adjustment. The Journal of Psychology, 150(4), 405-421. https:/ / doi.org/10.1080/002 23980.2015.1060185

Kökönyei, G., Józan, A., Morgan, A., Szemenyei, E., Urbán, R., Reinhardt, M., y Demetrovics, Z. (2015). Perseverative thoughts and subjective health complaints in adolescence: Mediating effects of perceived stress and negative affects. Psychology $\mathcal{E}$ Health, 30(8), 969-986. https:/ / doi.org/10.1080/08870446.2015.1007982

Jiang, W., Li, F., Jiang, H., Yu, L., Liu, W., Li, Q., y Zuo, L. (2014) Core Self-Evaluations Mediate the Associations of Dispositional Optimism and Life Satisfaction. PLOS ONE, 9(6): e97752. https:/ / doi.org/10.1371/journal.pone.0097752

Jibeen, T. (2013). Frustration intolerance beliefs as predictors of emotional problems in university undergraduates. Journal of Rational-Emotive $\mathcal{E}$ Cognitive-Behavior Therapy, 31, 16-26. http:/ / dx.doi.org/10.14349/sumapsi.2019.v26.n1.3

Landa, J. M. A., Luzón, M. D. C. A., y de Ugarte, M. F. S. (2008). El papel de la IEP y del optimismo/pesimismo disposicional en la resolución de problemas sociales: un estudio con alumnos de trabajo social. Electronic Journal of Research in Educational Psychology, 6(15), 363-382.

Linkins, M., Niemiec, R. M., Gillham, J., y Mayerson, D. (2014). Through the lens of strength: A framework for educating the heart. Journal of Positive Psychology. 10(1), 64-68. https:/ / doi. org/10.1080/17439760.2014.888581

McCullough, M. E., Emmons, R. A.c y Tsang, J. (2002). The grateful disposition: A conceptual and empirical topography. Journal of Personality and Social Psychology, 82, 112-127.

Marrero, R. J., y Carballeira, M. (2010). El papel del optimismo y del apoyo social en el bienestar subjetivo. Salud Mental, 33(1), 39-46.

Mayer, J. D., Caruso, D. R., y Salovey, P. (2016). The Ability Model of Emotional Intelligence: Principles and Updates. Emotion Review, 8(4), 290-300. https://doi. org/10.1177\%2F1754073916639667

Mayer, J. D., Salovey, P., y Caruso, D. (2004). Emotional Intelligence: Theory, findings and implications. Psychological Inquiry, 15(3), 197-215.

Mayer, J. D., y Salovey, P. (1997). What is emotional intelligence? En P. Salovey \& D. J. Sluyter (Eds.), Emotional development and emotional intelligence: Implications for educators (pp. 3-31). Basic Books.

Mustaca, A. E. (2018). Frustración y conductas sociales. Avances en Psicología Latinoamericana, 36(1), 65-81. http:/ /dx.doi. org/10.12804/revistas.urosario.edu.co/apl/a.4643

Oliva, A., Antolin, L., Pertegal, M., Rios, M., Parra, A., Hernando, A., y Reina, M. (2011). Instrumentos para la evaluación de la salud mental y el desarrollo positivo adolescente y los activos que lo promueven. Consejería de Salud.

Oliva, A., Pertegal Vega, M. Á., Antolín, L., Reina, M. D. C., Ríos, M., Hernando, Á., ... y Estévez, R. M. (2011). Desarrollo positivo adolescente y los activos que lo promueven: un estudio en centros docentes andaluces. Consejería de Salud.

Oliva, A., Ríos, M., Antolín, L., Parra, A., Hernando, A. y Pertegal, M. A. (2010). Más allá del déficit: Construyendo un modelo de desarrollo positivo adolescente. Infancia y Aprendizaje, 33(2), 223-234.

Ordóñez, A., González, R., Montoya-Castilla, I., y Schoeps, K. (2014). Conciencia emocional, estados de ánimo y rendimiento académico. International Journal of developmental and Educational Psychology. INFAD Revista de Psicología, 1(6), 229-236.

Ordóñez, A., Maganto, C., y González, R. (2015). Somatic complaints, emotional awareness and maladjustment in schoolchildren. Anales de Pediatría, 82(5), 308-315. https://doi.org/10.1016/j.anpede.2015.04.004
Parhiala, P., Torppa, M., Vasalampi, K., Eklund, K., Poikkeus, A., y Aro, T. (2018). Profiles of school motivation and emotional well-being among adolescents: Associations with math and reading performance. Learning and Individual Differences, 61, 196204. https:/ / doi.org/10.1016/j.lindif.2017.12.003

Peña-Sarrionandia, A., Mikolajczak, M. y Gross, J. J. (2015). Integrating emotion regulation and emotional intelligence traditions: A metaanalysis. Frontiers in Psychology, 6(160). https:/ / doi. org/10.3389/fpsyg.2015.00160

Perlman, S. B., Luna, B., Hein, T. C., y Huppert, T. J. (2014). fNIRS evidence of prefrontal regulation of frustration in early childhood. Neuroimage, 85(1), 326-334. https://doi.org/10.1016/j. neuroimage.2013.04.057

Pertegal, M. A., Oliva, A., y Hernando, A. (2010). Los programas escolares como promotores del desarrollo positivo adolescente. Cultura y Educación, 22, 53-66.

Peters, M. L., Meevissen, Y. M. C., y Hanssen, M.M (2013). Specificity of the Best Possible Selfintervention for increasing optimism: Comparison with a gratitude intervention. Terapia Psicológica, 31, 93-100.

Peterson, C., y Park, N. (2009). El estudio científico de las fortalezas humanas. En C. Vázquez y G. Hervás (coords.). La Ciencia del Bienestar. Fundamentos de una Psicología Positiva (pp. 181-207). Alianza Editorial.

Pulido, F., y Herrera, F. (2016). La inteligencia emocional como predictora del rendimiento académico infantil: el contexto pluricultural de Ceuta. Revista Complutense de Educación, 28, 1251-1265.

Pulido-Acosta, F., y Herrera-Clavero, F. (2019). Prediciendo el rendimiento académico infantil a través de la inteligencia emocional. Psicología Educativa, 25(1), 23-30. https://doi. org/10.5093/psed2018a16

Pulido-Acosta, F., y Herrera-Clavero, F. (2020). Estados emocionales contrapuestos e inteligencia emocional en la adolescencia. Psicología desde el Caribe, 37(1), 70-90.

Reina, C. R., y Oliva, A. (2015). From emotional competence to self-esteem and life-satisfaction in adolescents. Behavioral Psychology/Psicología Conductual, 23(2), 345-359.

Resurrección, D. M., Salguero, J. M., y Ruiz-Aranda, D. (2014). Emotional intelligence and psychological maladjustment in adolescence: a systematic review. Journal of Adolescence, 37(4), 461-472. https:/ / doi.org/10.1016/j.adolescence.2014.03.012

Rey, L., Extremera, N., y Pena, M. (2011). Perceived Emotional Intelligence, Self-Esteem and Life Satisfaction in Adolescents. Psychosocial Intervention, 20, 227-234. https://doi.org/10.5093/ in2011v20n2a10

Reyes, S., y Ferragut, M. (2016). Fortalezas psicológicas y diferencias de sexo en adolescentes. Escritos de Psicología (Internet), 9(3), 28-36. https:/ / doi.org/10.5231/psy.writ.2016.2811

Rodríguez, T. C., y Castañeda, M. C. (2015). Felicidad y optimismo en adolescentes y jóvenes peruanos y paraguayos: Un estudio predictivo. Salud E Sociedad, 6(3), 250-263. https://doi. org/10.22199/S07187475.2015.0003.00005

Rodríguez-Fernández, A., Ramos-Díaz, E., Ros, I., Fernández-Zabala, A., y Revuelta, L. (2016). Resiliencia e implicación escolar en función del sexo y del nivel educativo en educación secundaria. Aula Abierta, 44(2), 77-82. https:/ / doi.org/10.1016/j. aula.2014.05.004

Rodriguez, C. M., Russa, M. B., y Kircher, J. C. (2015). Analog assessment of frustration tolerance: Association with self-reported child abuse risk and physiological reactivity. Child Abuse $\mathcal{E} \mathrm{Ne}$ glect, 46, 121-131. https://doi.org/10.1016/j. chiabu.2015.02.017

Ruiz-Aranda, D., Cabello, R., Salguero, J. M., Palomera, R., Extremera, N., y Fernandez-Berrocal, P. (2013). Guía para mejorar 
la inteligencia emocional de los adolescentes. Programa INTEMO. Pirámide.

Rosenberg, M., y Owens, T. J. (2001). Personas con baja autoestima: un retrato colectivo. En T. J. Owens, S. Stryker y N. Goodman (Eds.), Ejecución de la teoría y la investigación de la autoestima: corrientes sociológicas y psicológicas (p. 400-436). https://doi. org/10.1017/CBO9780511527739.018

Ruvalcaba, N. A., Gallegos, J., Solís, O., Gabriela, M., y Bravo, H. R. (2019). Validez predictiva de las competencias socioemocionales sobre la resiliencia en adolescentes mexicanos. Diversitas: Perspectivas en Psicología, 15(1), 89-101. https://doi. org/10.15332/s1794-9998.2019.0015.07

Salovey, P., Mayer, J. D., Goldman, S. L., Turvey, C., y Palfai, T. P. (1995). Emotional attention, clarity, and repair: Exploring emotional intelligence using the Trait Meta-Mood Scale. En J. W. Pennebaker (dir.), Emotion, disclosure, and health (pp. 125-154). American Psychological Association.

Sánchez-Álvarez, N., Extremera, N., y Fernández-Berrocal, P. (2016). The relation between emotional intelligence and subjective well-being: a meta-analytic investigation. The Journal of Positive Psychology, 11, 276-285. https://doi.org/10.1080/174397 60.2015.1058968

Sánchez, Ó., y Méndez, F. X. (2009). El optimismo como factor protector de la depresión infantil y adolescente. Clínica y Salud, 20(3), 273-280.

Schoeps, K., Tamarit, A., González, R., y Montoya-Castilla, I. (2019). Competencias emocionales y autoestima en la adolescencia: impacto sobre el ajuste psicológico. Revista de Psicología Clinica con Niños y Adolescentes, 6(1), 51-56. https:/ /doi.org/10.21134/ rpcna.2019.06.1.7

Seligman, M. E. P. (2014). Florecer. La nueva psicología positiva y la búsqueda del bienestar. Editorial Océano.

Seligman, M. E. P., y Csikszentmihalyi, M. (2000). Positive psychology: an introduction. American Psychologist, 55, 5-14.
Sen, A., y Mukherjee, T. (2014). Personality traits, nature of aggression and frustration tolerance in children with conduct disorder. Indian Journal of Social Psychiatry, 30(1-2), 61-65.

Snyder, C. R. (2001). Coping with stress: Effective people and processes. Oxford University Press.

Thompson, H. M., Wojciak, A. S., y Cooley, M. E. (2016). Self-esteem: A mediator between peer relationships and behaviors of adolescents in foster care. Children and Youth Services Review, 66, 109-116. https://doi.org/10.1016/j.childyouth.2016.05.003

Vásquez, C. (2013). La psicología positiva y sus enemigos: Una réplica en base a la evidencia científica. Papeles del Psicólogo, 34(2), 91-115.

Vazquez, C., y Hervás, G. (2009). Psicología positiva. Desclée de Broker.

Ventura-León, J., Caycho-Rodríguez, T., Vargas-Tenazoa, D., y Flores-Pino, G. (2018). Adaptación y validación de la Escala de Tolerancia a la Frustración (ETF) en niños peruanos. Revista de Psicología Clínica con Niños y Adolescentes, 5(2), 23-29.

Villanueva, L., Montoya-Castilla, I., y Prado-Gascó, V. (2017). The importance of trait emotional intelligence and feelings in the prediction of perceived and biological stress in adolescents: Hierarchical regressions and fsQCA models. Stress, 20(4), 31-33. https: / / doi.org/10.1080/10253890.2017.1340451

von Soest, T., Wichstrøm, L., y Kvalem, I. L. (2016). The development of global and domain-specific self-esteem from age 13 to 31. Journal of Personality and Social Psychology, 110(4), 592-608. https:/ / doi.org/10.1037/pspp0000060

Wang, Y., Xie, G., y Cui, X. (2016). Effects of Emotional Intelligence and Selfleadership on Students' Coping with Stress. Social Behavior and Personality, 44(5), 853-864. https:/ / doi.org/10.2224/ sbp.2016.44.5.853

Yudes, C., Peña, L. R., y Pacheco, N. E. (2019). Ciberagresión, adicción a internet e inteligencia emocional en adolescentes: un análisis de diferencias de género. Voces de la Educación, 2, 27-44. 
